(95\% UI:-5000 to 39,000$), 32,000 \quad(95 \%$ UI: 9,500 to $56,000)$ and 17,000 (95\% UI: -7200 to 37,000$)$ respectively. Compared to the baseline, no scenario was more cost-effective nor reduced health inequalities; in all cases the probability of becoming cost-effective or equitable did not reach $80 \%$.

Conclusion Results suggest the intervention has the potential to reduce T2DM incidence but requires substantial participation and increased long-term effectiveness. The effects in other NCDs, cost-effectiveness and health inequalities were uncertain. Whilst reduction in T2DM is encouraging, a combination of high-risk and structural policies is needed to reduce the health inequalities gap and address the NCDs crisis, which is urgently overdue.

\section{OP12 PROJECTING THE BURDEN OF CARDIOVASCULAR DISEASE AND DIABETES IN GERMANY: NATIONAL TRENDS AND REGIONAL INEQUALITIES}

\footnotetext{
1,2Karl Emmert-Fees*, ${ }^{3}$ Shammi Luhar, ${ }^{4}$ Chris Kypridemos, ${ }^{1,2}$ Michael Laxy. ${ }^{1}$ Institute of Health Economics and Health Care Management, Helmholtz Zentrum München, Neuherberg, Germany; ${ }^{2}$ Associate Professorship of Public Health and Prevention, Technical University of Munich, Munich, Germany; ${ }^{3}$ Department of Public Health and Primary Care, University of Cambridge, Cambridge, UK; ${ }^{4}$ Department of Public Health and Policy, University of Liverpool, Liverpool, UK
}

\subsection{6/jech-2021-SSMabstracts.12}

Background Although age-adjusted mortality rates of coronary heart disease (CHD), stroke and type 2 diabetes mellitus (T2DM) are declining in many industrialised countries, the burden of these diseases may increase due to population aging. In Germany $33 \%$ of the population is expected to be older than 60 years by 2035 with differences between East and West Germany. Forecasting the burden and regional inequalities of $\mathrm{CHD}$, stroke and T2DM is vital for efficient health policy planning. This study aimed to 1) project mortality of CHD, stroke and T2DM by sex and age for the German population age 30 and older from 2018 to 2035 and 2) analyse regional inequalities in mortality between East and West Germany.

Methods We used population count estimates, cause-specific death counts (based on ICD-10-GM) from 1998 to 2018 and population projections to 2035 provided by the German Federal Statistical Office. Cause-specific mortality rates were forecast until 2035 for each sex, region and 5-year age category using a functional demographic model. The model was calibrated using a root mean squared error approach based on the last five observed years of data and validated graphically. Uncertainty was computed as 95\%-confidence intervals (CI). Age-sex-standardised mortality rate ratios (MRRs) between East and West Germany were estimated using direct standardisation with the 2018 German population as the standard population. Projected death counts were calculated by multiplying mortality rates with projected population counts. We used R v4.0.3 with the packages demography v1.22 and forecast v8.13.

Results We found that annual mortality from CHD in Germany is projected to decrease by $36.52 \%$ ( 23200 deaths, 95\%-CI: 14400-30800) for men and 31.70\% ( 22400 deaths, 95\%-CI: 10000-32200) for women by 2035. Mortality from stroke and T2DM is projected to increase by $21.03 \% \quad(\sim 6200$ deaths, 95\%-CI: -15000-59300) and $29.68 \%$ ( $\sim 4200$ deaths, 95\%-CI: -1200-12000) for men and decrease by $40.30 \%$ ( 16300 deaths, 95\%-CI: 11600-
20200) and 14.03\% ( 2300 deaths, 95\%-CI: -3700-6500) for women, respectively. Age-sex-standardised MRRs show that mortality from these causes is higher in East Germany. By 2035, inequalities are projected to narrow for CHD and T2DM, the latter potentially being reversed, and remain constant for stroke. The model performed well in validation analyses.

Conclusion Our projections suggest considerable future decreases in CHD mortality for both men and women in Germany. Deaths from stroke and T2DM are projected to increase for men, while decreasing among women. Inequalities between East and West Germany are expected to decline but largely persist throughout the projection period.

\section{Wednesday 15 September Children, $13.00-15.25$}

\section{OP13 EARLY CHILD DEVELOPMENT AT 2-5 YEARS PREDICTS COGNITIVE OUTCOMES AT 6-9 YEARS IN LAO PDR: A CASE FOR POPULATION MONITORING USING THE EARLY HUMAN CAPABILITY INDEX IN LOW AND MIDDLE INCOME COUNTRIES}

\footnotetext{
${ }^{1,2}$ Alanna Sincovich*, 1,2Tess Gregory, 1,3 John Lynch, ${ }^{1,2}$ Sally Brinkman. 'School of Public Health, University of Adelaide, Adelaide, Australia; ${ }^{2}$ Telethon Kids Institute, University of Western Australia, Adelaide, Australia; ${ }^{3}$ Population Health Sciences, Bristol Medical School, University of Bristol, Bristol, UK
}

\subsection{6/jech-2021-SSMabstracts. 13}

Background Beyond effects of linear growth on cognitive development, research has seldom focused on children's developmental trajectories in low and middle income countries. This is limited by a lack of suitable measurement tools. Global commitment to tracking early child development, as outlined by the Sustainable Development Agenda, has spurred efforts to address this challenge. The early Human Capability Index (eHCI) has been shown to be a feasible and valid population monitoring measure across diverse contexts. This study investigated the comparative ability of the eHCI and direct assessment of children's development at 2-5 years in predicting cognitive outcomes at 6-9 years.

Methods We used data collected as part of the Early Childhood Education Project, financed by the World Bank Group, in Lao PDR. Baseline data collected commenced in 2015. The sample was drawn from 376 villages in Northern Laos selected on the basis of poverty level. In each villages, 20 random households with at least one child aged between 2-5 years were selected. In 2020, children within the age range of 6-9 were surveyed again. The study population included all children for whom data were collected at both time points $(n=5,269)$. Four measures of children's development were used in this study; eHCI overall development, and direct assessment literacy, numeracy, and executive function. The eHCI, collected via caregiver report, includes 56 items designed to measure early child development across 8 domains. Children's literacy, numeracy, and executive function were measured via 92 direct assessment items. Receiver Operating Characteristic (ROC) curves, Cstatistics and $95 \%$ confidence intervals were estimated to determine the ability of scores at time 1 to predict poor scores at time 2 . 